\title{
Daycare Experiences and Long-Term Behavioral Outcomes: A Retrospective Self-Report
}

\author{
Amy R. Murrell", Jeffrey D. Geddes, Emily Yancey, Karen M. O’Brien and Francis Terrell
}

Psychology Department, University of North Texas, Denton, TX 76203-1280, USA

\begin{abstract}
The current study examined existing data to determine whether family SES, age of entry into daycare, time spent in daycare per week, and child personality variables predicted behavioral outcomes. Our analyses indicated that, for this sample, the best predictor of problem behavior was personality. Specifically, individuals low in agreeableness reported more problem behavior than did individuals high in agreeableness. Family SES, age of entry into daycare, and amount of time spent in daycare were not significantly related to problem behavior. Although daycare quality was significantly correlated with problem behavior, it only accounted for $2 \%$ of the variance. Such findings indicate that future researchers should examine mediating or moderating effects of personality on the relationship between daycare and behavior.
\end{abstract}

Keywords: Daycare quality, behavioral outcomes, retrospective self-report.

Daycare has become an integral part of the lives of many families and children [1]. In the year 2000, in the United States, $80 \%$ of children under age six were spending an average of 40 hours per week in some form of non-parental care [2]. Other reports indicate that over $50 \%$ of children of all ages are spending at least some of their time in nonparental child care settings [3-5]. With such a large percentage of children spending a significant amount of time in nonparental care settings it is important to explore the impact of these child-care experiences on child development and behavior.

This study, therefore, examined the relationship between self-report of critical behavioral outcomes of adolescence (i.e., school suspensions and imprisonment) and daycare variables, including age of entry and length of time in daycare as well as daycare quality. Several family and personality characteristics were examined as well. These factors were chosen for investigation based on a review of the daycare literature. This literature will be briefly summarized prior to introduction to the current project.

\section{VARIABLES AFFECTING OUTCOMES}

Numerous studies have explored the differences in development and behavior of children who have spent time in non-parental care settings. Research has revealed that childcare in general can have positive implications for social and emotional development, behavior, cognitive abilities, and assertiveness $[1,3,6]$. Studies have indicated several variables of non-parental care settings that influence the differences in children's behavior and development. These include: age of entry into care, time spent in care, family characteristics, and quality of the care. Each of these variables will be discussed in more detail.

\footnotetext{
*Address correspondence to this author at the Psychology Department, University of North Texas, P.O. Box 311280, Denton, TX 76203-1280, USA; Tel: 940-565-2967; Fax: 940-565-4682; E-mail: amurrell@unt.edu
}

\section{Age of Entry}

The developmental and behavioral impact of age of entry into childcare has been debated in the literature. Results from the NICHD Study of Early Child Care [6] indicated that age of entry into childcare did not significantly predict child socioemotional development and behavioral outcomes. Additionally, Howes [7] found that enrolling children in daycare after infancy was not related to the later development of problem behaviors, and Andersson [4] found that children entering daycare at an early age both performed better on cognitive tests and received better ratings of social-personal attributes from teachers. In contrast, Maccoby and Lewis [8] found that more hours spent in non-parental care during the first four and a half years of life increased a child's propensity toward problem behavior and decreased social competence. The evidence presented in literature up to this date has been inconclusive about the impact on a child due to the age at which he or she was placed in daycare.

\section{Time}

The amount of time spent in a daycare setting has also provided researchers with mixed results concerning children's developmental outcomes. The NICHD Study of Early Child Care [6] found that the amount of time that a child spends in non-parental childcare in the first two to three years of life was not significantly related to self-control, compliance or problem behavior at a later age. However, in 2006, results from the longitudinal NICHD Study of Early Child Care Research Network [9] found that increased time spent in childcare per week was associated with increased problem behaviors, teacher-child conflict, and decreased social skills at the age of 4.5 years.

\section{Quality}

There is no uniform system that assesses the quality of non-parental care settings; therefore, daycare quality has been measured in a variety of ways. Various study designs have used the Early Childhood Environment Rating Scale 
(ECERS) as a tool to evaluate the quality of day care settings. The ECERS is a 43-item questionnaire that assesses the quality of a childhood environment including; personal care routines, space and furnishings, language reasoning, activities, interactions, program structure, and parents and staff [10]. Others have used a combination of visiting day care settings and interviewing caregivers to evaluate the quality of the care setting [9,11]. Marshall [2] defined quality in terms of structural and process indicators. Structural characteristics include staff ratio, day care group size, and education of specialized day care teachers and directors. Process characteristics refer to the nature of care that children experience (e.g., warmth, sensitivity, responsiveness of caregivers, activities available and the developmental appropriateness of those activities). Marshall defined a high quality childcare setting as one that supports optimal learning and development. Most of the literature uses some combination of structural and/or process variables in their evaluations of day care quality.

Quality of non-parental care has historically been one of the best predictors of childhood outcomes. Higher quality childcare has been associated with positive social, behavioral and cognitive outcomes [2-4, 8, 11-13]. The NICHD Study of Early Child Care [9] found that children who experienced higher quality care scored higher on cognitive measures, ratings of social and peer outcomes and language skills. In 1996, the NICHD [14] also found that higher quality of care in the first two years of life predicted fewer social problems and more compliance with parents/guardians and nonparental figures. Child care quality has also been positively linked to social development and competence, emotional security, cognitive and verbal abilities, and decreased behavioral problems $[3,7,13,15,16]$.

\section{Family Characteristics}

Family characteristics independently serve as significant predictors of child outcome, although they are often related to quality and time spent in daycare. The NICHD study revealed that parenting served as a large predictor of child outcomes. Children who experienced more responsive and stimulating interactions with parents displayed better cognitive, language and socioemotional development [9]. Marshall [2] found that positive associations between childcare quality and children's school performance were stronger for mothers who had less education. Hagekull \& Bohlin [11] also found that high quality daycare contributed to the diminution of externalizing behaviors for children living in homes with lower quality factors, such as a messy, unstructured, and cold environment; however, for children from high or medium quality homes, daycare quality did not offer an explanation for variation in behavior. Socioeconomic status (SES) of families also contributes to various outcomes. Low SES families tend to send their children to lower quality day care centers and these children also tend to spend more time in non-parental care. Children from lower SES families subsequently demonstrate increased behavioral problems and lower cognitive and social abilities $[1,9,11,15]$.

\section{BIG-5 VARIABLES}

Personality variables can also play a part in determining child social and behavioral outcomes. The five-factor model also known as the Big-Five theory [17-19] consists of five distinctive traits, which include: Neuroticism, Extraversion, Openness to Experience, Agreeableness, and Conscientiousness [20]. This model is data driven and applicable to a nonclinical general population, and thus it is widely accepted as a solid way to assess personality characteristics.

Individuals who score high in neuroticism not only experience negative affect such as fear, sadness, embarrassment, anger and guilt at high levels, but they are also more susceptible to the effects of this psychological distress. High scorers in neuroticism are often less able to control impulses and cope appropriately with stress. Extraversion refers to the tendency to be outgoing, assertive, active and talkative. Openness is associated with the propensity to be curious about both the inner and outer world. Agreeableness refers to the tendency to be altruistic, sympathetic and helpful. Conscientiousness is the general ability to resist impulses and temptations. Individuals high in conscientiousness are characterized by compulsive neatness, determination and being strong willed [20].

Research has shown some correlations between some of these factors and behavioral, social and emotional patterns in children and adolescents. Gleason, Jensen-Campbell and Richardson [21] examined the relationship between aggression and agreeableness in adolescence. They found that adolescents who are more agreeable tend to strive for affiliation in social situations and desire to maintain positive relationships with others. Adolescents who rated highly in the agreeableness category were found to have a low frequency of aggressive behaviors and aggressive cognitions. Other research has found that lower levels of conscientiousness, openness to experience and agreeableness were associated with poor social behavior and externalizing behaviors such as attentional deficits, conduct problems and hyperactivity. Neuroticism has also been associated with social difficulties and internalizing problems such as depression and anxiety $[20,22]$. There has been little research examining the correlation between external childcare settings such as day care settings and long-term outcomes associated with personality variables. One study, however, found a relation between an extraverted personality and attendance in a day care setting. The same study found that more daycare early in life predicted emotional stability [23]. Further research is needed to examine the effects of day care on child and adolescent personality.

\section{CURRENT STUDY}

The current study examined an existing data set of factors related to daycare, previously designed and collected by two of the current authors (EY \& FT). Initial analyses conducted with the data set gave rise to several additional research questions. The aim of this study was to answer some of those questions; specifically, to determine whether family SES, age of entry into daycare, time spent in daycare per week, and personality variables predicted future behavioral outcomes for that sample. In light of the variable nature of the state of literature, the most recent findings regarding age of entry into daycare [8] and the 2006 NICHD [10] findings on time spent in daycare guided the hypotheses of the current study. It was hypothesized that report of low SES, a younger age of entry into daycare, and more hours spent in daycare per week would be associated with more negative behavioral 
outcomes in the future. Additionally, given that a variety of research has shown agreeableness to be an important factor in behavioral outcomes, participants low on agreeableness were expected to report more negative future behavioral outcomes. No specific hypotheses were made with respect to other personality factors.

The specific methodology used for creating the variables for these analyses were based on several factors, two of them being particularly relevant to result interpretation. First, analyses on the data for this paper were conducted secondarily to a larger study. Second, given that the literature review revealed no gold standard for evaluating daycare quality or long-term outcomes, the researchers' clinical judgment was utilized to define these variables. It should be noted that the manner in which the independent and dependent variables were assessed and then quantified for analyses are, thus, unique to this study.

To date, research that examines the relationship between long-term effects of childhood daycare factors and outcomes during late adolescence is scarce. In contrast, most research has examined outcomes of early childcare at three and five years of age. Research has not yet examined how a child's subjective experience of the quality of a daycare setting might correlate with future developmental and behavioral outcomes. As such, the current study examined the relationship between a child's subjective experience of day care quality and future behavioral outcomes as well as examined the long-term effects of early childcare on later outcomes.

\section{METHOD}

As previously stated, the current research question evolved from initial analyses of a larger data set on daycare factors. The original project was designed and carried out in the year prior to the analyses conducted for this paper. Initial analyses did not address behavioral outcomes. Thus, the authors designed a research question about such outcomes based on what was available in the data set that had not already been examined. The subset of data was cleaned and then tested to ensure that all assumptions of various tests were met before running the current analyses. Descriptions of participants and measures refer to the relevant features of the initial study.

\section{Participants}

Participants were recruited from an undergraduate population at a large state university in the southwestern United States. Sign up sheets were placed in the Psychology Building on campus and the questionnaires were administered to students in groups of 5 to 25 persons at a time. Prior to participation, all participants were provided a consent form and given the opportunity to ask questions about the study, which was IRB approved. The sample consisted of 100 participants (80 women and 20 men). Their mean age was 20.95 years, and $88 \%$ were traditional students in that they had come right out of high school into college. There was very little variation in the time since they had been in daycare.

\section{Measures}

Background Information Questionnaire. A Background Information Questionnaire was created specifically for this study. The last item of this questionnaire, "Category in which your family's annual gross income falls," was used as a measure of family SES. Response options included (1) Less than $\$ 5000,(2), \$ 5000-\$ 25,000$, (3) $\$ 25,001-\$ 50,000$, (4) $\$ 50,000-\$ 75,000,(5), \$ 75,001$ to $\$ 100,000$ and (6) over $\$ 100,000$.

Daycare questionnaire. A daycare questionnaire was also created specifically for this study. This self-report questionnaire examined how much time the participant spent in daycare as a child and how they felt about their daycare experience. Some parents also completed the measures, reporting on their children's daycare experiences. The questionnaire included items designed to assess problem behavior that the participant may have engaged in later in adolescence. Age of entry into daycare was assessed with the question, "At approximately what age were you placed in Daycare?" Response options included (1) I was never placed in Daycare, (2) at about 6 weeks of age, (3) at about 6 months of age, (4) at about one year of age, and (5) when I was over one year of age. Time spent in daycare per week was assessed with the question, "On average, how many days per week were you in Daycare?" Response options included (1) about one day per week, (2) about two days per week, (3) about three days per week, (4) about four days per week, (5) about five days or more per week. In addition, a set of similarly scaled items assessed problem behaviors such as being arrested or suspended from school.

Based on clinical judgment, both a daycare quality and total problem behavior variable were created by combining relevant questions from the Daycare Questionnaire. Specifically, items measuring participants' perceptions of how they were treated in daycare were summed to create the daycare quality variable, and items measuring participants' problem behavior, including school suspensions and jail time, were summed to create the problem behavior variable.

Big Five Inventory (BFI )[24]. This questionnaire was administered to assess neuroticism, extraversion, openness, conscientiousness, and agreeableness. The BFI consists of 44 descriptive phrases, with 8 to 10 phrases to assess each trait. This measure has been shown to have adequate or better internal reliability (.7 or higher) consistency in several samples.

\section{RESULTS}

Normality of variables was checked and all were found to be appropriate for analysis. Descriptive statistics were calculated for all predictor variables and for the criterion variable. The means and standard deviations for family income (the Family SES), age of entry into daycare, and days per week in daycare were calculated and are represented in Table 1.

For ease of interpretation, prior to conducting any statistical analyses the agreeableness and quality variables were reverse coded, so that lower scores on both measures would indicate higher levels of both agreeableness and quality. As can be seen in Table 2 , a correlation matrix revealed significant correlations between the predictor variables Family SES and Quality $(p<.01)$ as well as between Days per Week and Agreeableness $(p<.01)$. Additionally, both Quality and Agreeableness were significantly correlated with the 
criterion variable, Total Problems ( $p<.05$ and $p<.01$ respectively). The correlations between predictor variables did not violate the assumptions of non-collinearity, nor multicollinearity.

Table 1. Descriptive Statistics for Predictor and Criterion Variables

\begin{tabular}{|c|c|c|}
\hline \multirow{2}{*}{ Variable } & Mean & SD \\
\hline & \multicolumn{2}{|c|}{ Participants $(n=100)$} \\
\hline 1. Family SES & 3.54 & 1.37 \\
\hline 2. Age of entry & 4.41 & .96 \\
\hline 3. Days per week & 3.92 & 1.412 \\
\hline 4. Quality & 7.49 & 2.93 \\
\hline 5. Agreeableness & 24.53 & 5.59 \\
\hline 6. Total problems & 4.27 & 1.73 \\
\hline
\end{tabular}

Table 2. Intercorrelations Among Predictor and Criterion Variables

\begin{tabular}{|l|c|c|c|c|c|c|}
\hline \multirow{2}{*}{ Variable } & $\mathbf{1 .}$ & $\mathbf{2 .}$ & $\mathbf{3 .}$ & $\mathbf{4 .}$ & $\mathbf{5 .}$ & $\mathbf{6 .}$ \\
\cline { 2 - 7 } & \multicolumn{7}{|c|}{ Participants $(\boldsymbol{n}=\mathbf{1 0 0})$} \\
\hline \hline 1. Family SES & - & -.11 & -.01 & $-.25^{* *}$ & -.09 & -.13 \\
\hline 2. Age of entry & - & -.19 & .10 & -.02 & .09 & \\
\hline 3. Days per week & - & -.06 & $.26^{* *}$ & .08 & & \\
\hline 4. Quality & - & .10 & $.18^{*}$ & & & \\
\hline 5. Agreeableness & - & $.29^{* *}$ & & & & \\
\hline 6. Total problems & - & & & & & \\
\hline$* p<.05 * * p<.01$. & & & &
\end{tabular}

A multiple regression analysis was conducted to evaluate the relative contributions of family income, age of entry into daycare, time spent in daycare per week, daycare quality, and agreeableness in the variance of the Total Problems variable (reported school suspensions and jail time). The linear combination of these predictor variables was significantly related to Total Problems, $F(5,115)=3.47, p<.01$. The sample multiple correlation coefficient $\left(R^{2}\right)$ was $.13(95 \%$ confidence interval $(\mathrm{CI})=.002-.234)$, indicating that approximately $13 \%$ of the variance in Total Problems for the sample can be accounted for by the linear combination of family income, age of entry into daycare, time spent in daycare per week, daycare quality, and agreeableness.

Indices that indicate the relative strength of the individual predictors can be found in Table 3 . The relationship between agreeableness and total problems was significant, $t=2.57, p$ $=.01$, and agreeableness accounted for $6 \%$ of the variance in total problems for the sample. Daycare quality (as measured by self-report of treatment in daycare) accounted for $2 \%$ of the variance in total problems for the sample.

\section{DISCUSSION}

Our analyses indicated that, for this sample, the best predictor for future problem behavior was agreeableness $(p<$ $.05)$, such that individuals low in agreeableness reported more problem behavior than did individuals high in agreeableness. This finding supports the research of Gleason, Jensen-Campbell, and Richardson [21]. However, research findings suggesting that family income, age of entry into daycare, and amount of time spent in daycare per week were related to child/adolescent problem behavior were not supported $[1,8,9,11,15]$. The linear combination of family income, age of entry and days per week accounted for less than $1 \%$ of the variance in future problem behavior in the current sample.

Table 3. Relative Strength of Individual Predictors $(n=100)$

\begin{tabular}{|c|c|c|c|c|}
\hline & $\boldsymbol{\beta}$ & $\boldsymbol{t}$ & $\boldsymbol{p}$ & $\boldsymbol{R}^{2}$ \\
\hline \hline Family SES & -.06 & -.60 & .55 & .00 \\
\hline Age of entry & .08 & .83 & .41 & .01 \\
\hline Days per week & .04 & .34 & .74 & .00 \\
\hline Quality & .14 & 1.36 & .18 & .02 \\
\hline Agreeableness & .26 & $2.57^{*}$ & .01 & .06 \\
\hline$p<.05$.
\end{tabular}

Although daycare quality was significantly correlated to future problem behavior for this sample, it only accounted for $2 \%$ of the variance in future problem behavior. Because agreeableness accounted for more variance than did quality, future researchers should examine possible mediating or moderating effects of agreeableness and other personality variables that might influence the relationship between daycare quality and problem behavior.

\section{Limitations of the Current Study}

Notably, the current study suffered from significant problems with reliable and valid measurement. First and perhaps most importantly, measurement of both daycare quality and problem behavior should be conducted using more reliable measures. The quality and problem behavior variables created in this study were limited by the nature of the items in the Daycare Questionnaire. Daycare quality undoubtedly involves more than adult retrospective accounts of how they were treated as children, and problem behavior defined solely as school suspensions and jail time is a very limited concept. It is also recognized that suspensions and jail time are perhaps falsely measured as singular incidents. School suspensions may have resulted after a number of warnings or other academic punishments, and jail time may have been avoided even in cases of youth who have had several arrests. In addition, it is possible that recollection of early life experiences was distorted, especially since the average age of daycare entry was approximately 17 years prior to the average age of the current sample. It should be noted, however, that there was no clear or significant disagreement about daycare factors or problem behaviors found between participant response and their parents' in the 48 cases where 
parental response was available. Even if memories were wholly accurate, however, data would still have been lost due to the conversion of family SES, age of entry into daycare, and time spent in daycare into categorical variables. Finally, with respect to measurement, assessing family SES in the present may not have been ideal. A more sophisticated approach would be to consider SES at both entry into daycare as well as at time of retrospective report, as well as any changes over time and their relationship to problem behavior. An additional limitation is that the sample may not be generally representative of children in daycare, or even adults who were in daycare as children. All participants were college students, and most were female; this sample may be biased, as being educated and being female are both protective factors when it comes to the behavioral outcomes selected.

\section{Future Directions}

Daycare quality needs to be more objectively assessed. Future work on this topic would be greatly advanced by the use of set criteria. A measure that summarizes quality variables such as caregiver-child ratio, types of resources and materials available, and curriculum successes would be ideal. Using this measure when students are in daycare, as opposed to relying on their self-report of such factors would also strengthen investigations like this. Likewise, obtaining school and juvenile justice records would be better than using self-report of negative behavioral outcomes. These records would eliminate the self-report bias and could be used to measure the entire continuum of problem behavior in a more detailed way. Future researchers should measure family SES (both past and present), age of entry into daycare, and time spent in daycare per week as continuous variables rather than providing participants with somewhat arbitrarily defined discrete response options. With more reliable and valid measures of both family and daycare variables affecting later behavioral outcomes, as well as more reliable and valid measures of the behavioral outcomes themselves, researchers can hope to develop a more accurate model of the predictive power of such variables on childhood development and problem behavior later in life. It should be noted that few articles on daycare outcomes have been written in recent years, and this is clearly an area of work that needs to be developed.

\section{Implications}

Despite the measurement limitations present in the current study, the implications of these results for parents who utilize daycare services are promising. Most notably, the assumption that non-parental childcare is more detrimental than not seems to be a misguided assumption. For this sample at least, children from families of lower SES who entered daycare at an earlier age and who spent more time in daycare did not evidence any more future problem behavior than did children from families of higher SES who entered daycare later and spent less time there. Though in some studies these variables have shown an impact on the development of problem behavior, the current study suggests that children of low SES homes who enter daycare early and spend a significant amount of time in daycare will not necessarily evidence negative behavioral outcomes.

It is clear that the factors predicting childhood development problems and negative behavioral outcomes are many, and the relationships among them are complex. As noted, research into the variables affecting outcome of non-parental childcare has produced contradictory and inconclusive findings. It is not unlikely that these mixed findings, along with the unexpected results of this study, indicate that our understanding of the issue is incomplete and/or that the issue is truly as complicated as the findings suggest.

Further studies, conducted with more representative samples and more precise measurement, are crucial.

\section{REFERENCES}

[1] DiLalla LF. Daycare, child, and family influences on preschoolers' social behaviors in a peer play setting. Child Study J 1998; 28: 22344.

[2] Marshall N. The quality of early child care and children's development. Early Child Dev Care 2004; 13: 165-8.

[3] Phillips D, McCartney K, Scarr S. Child-care quality and children's social development. Dev Psychol 1987; 23: 537-43.

[4] Andersson BE. Effects of public day care: a longitudinal study. Child Dev 1989; 60: 857-66.

[5] Shpancer N, Bennett-Murphy L. The link between daycare experience and attitudes toward daycare and maternal employment. Early Child Dev Care 2006; 176: 87-97.

[6] NICHD Early Child Care Research Network. Early child care and self-control, compliance, and problem behavior at twenty-four and thirty-six months. Child Dev 1998; 69: 1145-70.

[7] Howes C. Can the age of entry into the child care and the quality of child care predict adjustment in kindergarten? Dev Psychol 1990; 26: 292-303.

[8] Maccoby E, Lewis C. Less day care or different day care? Child Dev 2003; 74: 1069-75.

[9] NICHD Early Child Care Research Network. Child- care effect sizes for the NICHD study of early child care and youth development. Am Psychol 2006; 61: 99-116.

[10] Warash B, Markstrom C, Lucci, B. The early childhood environment rating scale-revised as a tool to improve child care centers. Education 2005; 126: 240-50.

[11] Hagekull B, Bohlin G. Day care quality, family and child characteristics and socioemotional development. Early Child Res Q 1995; 10: 505-26.

[12] Field T. Quality infant day-care and grade school behavior and performance. Child Dev 1991; 62: 863-70.

[13] Howes C, Phillips D, Whitebook M. Thresholds of quality: implications for the social development of children in center-based child care. Child Dev 1992; 63: 449-60.

[14] NICHD Early Child Care Research Network. NICHD study of early child care and youth development. Early Child Res Q 2006; 11: 269-306.

[15] Broberg A, Wessels H, Lamb M, Hwang C. Effects of day care on the development of cognitive abilities in 8-year-olds: a longitudinal study. Dev Psychol 1997; 33: 62-9.

[16] Pierrehumbert B, Ramstein T, Karmaniola A, Miljkovitch R, Halfon O. Quality of child care in preschool years: a comparison of the influence of home care and day care characteristics on child outcome. Int J Behav Dev 2002; 26: 385-96.

[17] Costa Jr PT, McCrae RR. Four ways five factors are basic. Pers Individ Dif 1992; 13: 653-65.

[18] Costa Jr PT, McCrae RR. Reply to Eysenck. Pers Individ Dif 1992; 13: 861-5.

[19] Goldberg LR. An alternative description of personality: the big-five factor structure. J Pers Soc Psychol 1990; 59: 1216-29.

[20] Ehrler D, Evans J, Mcghee R. Extending big-five theory into childhood: a preliminary investigation into the relationship between bigfive personality traits and behavior problems in children. Psychol Sch 1999; 36: 451-8. 
[21] Gleason K, Jensen-Campbell L, Richardson D. Agreeableness as a predictor of aggression in adolescence. Aggress Behav 2004; 30: 43-61.

[22] Martin R. Are behavioral problems in preschool children related to big-five markers. In: Proceeding of the Biennial Meeting of the Society for Research in Child Development, New Orleans, LA 1993.
[23]

Hagekull B, Bohlin G. Preschool temperament and environmental factors related to the five-factor model of personality in middle childhood. Merrill Palmer Q 1998; 44: 194-215.

[24] John OP, Srivastava S. The big five trait taxonomy: history, measurement, and theoretical perspectives. In: Pervin LA, Ed. Handbook of personality: theory and research. New York: Guilford Press 1999; pp. 102-38.

(C) Murrell et al.; Licensee Bentham Open.

This is an open access article licensed under the terms of the Creative Commons Attribution Non-Commercial License (http://creativecommons.org/licenses/by$\mathrm{nc} / 3.0 /$ ) which permits unrestricted, non-commercial use, distribution and reproduction in any medium, provided the work is properly cited. 